\title{
NOTA
}

\section{TRAGUS AUSTRALIANUS (POACEAE, CYNODONTEAE), NUEVO REGISTRO DE UNA ESPECIE EXÓTICA PARA EL PARAGUAY}

\author{
JUANA DE EGEA ${ }^{1,2}$, FÁTIMA MERELES ${ }^{1,2} \&$ GLORIA CÉSPEDES ${ }^{1,2,3}$
}

\begin{abstract}
Summary: De Egea, J., F. Mereles \& G. Céspedes. 2016. Tragus australianus (Poaceae, Cynodonteae), new record of an exotic species for Paraguay. Bonplandia 25(1): 73-77.
\end{abstract}

Tragus australianus S. T. Blake is cited for the first time for the Paraguayan flora. A brief description of the species, herbarium specimen image and comments on its ecology and geographic distribution is presented.

Key words: Cynodonteae, exotic species flora, Paraguay, Tragus.

Resumen: De Egea, J., F. Mereles \& G. Céspedes. 2016. Tragus australianus (Poaceae, Cynodonteae), nuevo registro de especie exótica para el Paraguay. Bonplandia 25(1): 73-77.

Tragus australianus S. T. Blake se cita por primera vez para la flora paraguaya. Se presenta una breve descripción de la especie, imagen del espécimen de herbario y comentarios sobre su ecología y distribución geográfica.

Palabras clave: Cynodonteae, especie exótica, flora, Paraguay, Tragus.

\section{Introducción}

El género Tragus Haller, anteriormente circunscripto dentro de la tribu Zoysieae (Clayton \& Richardson, 1973) y actualmente dentro de la tribu Cynodonteae, subtribu Traginae (Peterson et al., 2010; Soreng et al., 2015), está compuesto por ocho especies (Clayton et al., 2014) de las cuales siete son nativas de los trópicos y subtrópicos del Viejo Mundo y de Australia (Anton, 1981) y una de Argentina, Tragus andicola Zapater \& Sulekic (Sulekic \& Zapater, 2001).
Tres especies de Tragus fueron introducidas en América y son conocidas por encontrarse con frecuencia como malezas de zonas secas, tropicales y subtropicales. Entre ellas, la más común es $T$. berteronianus Schult., ampliamente distribuida desde el suroeste de Estados Unidos de América hasta la Argentina (Anton, 1981). Sulekic \& Zapater (2001) mencionan que en la Argentina es una especie muy frecuente en suelos arenosos y pedregosos, a menudo perturbados. En el Paraguay, T. berteronianus era el único

\footnotetext{
${ }^{1}$ Investigador Asociado del Centro para el Desarrollo de la Investigación Científica. Fundación Moisés Bertoni para la Conservación de la Naturaleza y Laboratorios Díaz-Gill, Manduvirá 635, Asunción, Paraguay. E-mail: juana. deegea@gmail.com

${ }^{2}$ Investigador del Programa Nacional de Incentivo a Investigadores del Consejo Nacional de Ciencia y Tecnología (CONACYT).

${ }^{3}$ Asociación Etnobotánica Paraguaya, Julia Miranda Cueto de Estigarribia 795, Edificio Mariucci, $2^{\circ}$ piso, oficina 208, San Lorenzo, Paraguay.
} 
representante del género registrado hasta la fecha; aunque la especie sólo es conocida por dos registros de herbario colectados en el Jardín Botánico de Asunción, y en el Departamento Boquerón (base de datos TROPICOS.org, 2015).

En colecciones recientes realizadas en el Chaco paraguayo, específicamente en zonas de cultivos, fue registrada por primera vez para el país la especie T. australianus S. T. Blake, nativa de Australia e introducida en Europa y Sudamérica. Se presenta una breve descripción de la especie y comentarios sobre su ecología y distribución.

Tragus australianus S. T. Blake, University of Queensland Papers, Department of Biology 1(18) 1941. Typus: Australia. Queensland: Maranoa District, Noondoo near Dirranbandi, 4-III-1936, S. T. Blake 10660 (holotypus, BRI, isotypi, CANB, K, MEL). Fig. 1.

Hierbas anuales, erectas o ascendentes, de $20-40 \mathrm{~cm}$ de altura. Cañas erectas. Vainas abiertas. Lígulas 0,5-1 mm, pilosas. Láminas 2-10 × 0,3-0,5 cm, planas, rígidas, acuminadas, glabras, glaucas; márgenes tuberculado-ciliados; cilios de hasta 1,5 mm. Inflorescencias de 5-8 $\mathrm{cm}$, subespiciformes; raquis estriado y piloso; fascículos numerosos, apretados, caedizos. Espiguillas pareadas, fértiles; pedúnculos de 0,5$1 \mathrm{~mm}$, con pelos hialinos, uncinados; espiguilla basal de 3-4,5 mm, subsésil; espiguilla distal de igual longitud o ligeramente menor, separada de la primera por un entrenudo de $0,5 \mathrm{~mm}$. Gluma inferior de 0,3-0,5 mm, lanceolada, hialina; gluma superior de 3-4 mm, ovada, acuminada, coriácea, 5-nervada, con pelos uncinados de base tuberculada sobre los nervios y pubescencia breve entre las venas. Lemma de 2,5-4 mm, ovada, acuminada, hialina, 3-nervada. Pálea 1,82,2 mm, hialina, 2-aquillada, ápice denticulado. Lodículas 2, cuneadas, hialinas. Estambres 3. Cariopsis 1,5-1,8 mm long.

Obs.: Tragus australianus está estrechamente relacionada con $T$. berteronianus, de la cual se diferencia principalmente por caracteres cuantitativos (Anton, 1981), relacionados con: 1) la longitud de las espiguillas (3-4,5 mm, ambas de igual longitud o la superior poco menor, ambas fructíferas vs. la inferior de $(1,6)$ 2-2,8 $(-3,2) \mathrm{mm}$, fructífera y la superior de $(0,8) 1-2,5$ (-3) mm, fructífera o estéril) y 2) el pedúnculo del fascículo $(0,5-1 \mathrm{~mm}$ generalmente mayor que la longitud del entrenudo que separa ambas espiguillas vs. 0,2-0,5 mm, menor o igual que la longitud del entrenudo).

Distribución geográfica y ecología: Especie nativa de Australia, introducida en Europa y Sudamérica. Según Zuloaga \& Belgrano (2014), se atribuye a esta especie el estatus de adventicia. En Argentina ha sido registrada en las provincias La Pampa, La Rioja, San Juan, San Luis (Zuloaga \& Belgrano, 2014), Salta y Formosa (Sulekic \& Zapater, 2001). En este trabajo se reporta por primera vez la presencia de Tragus australianus en Paraguay (Fig. 2), registrada en el Dep. Boquerón, limítrofe con las provincias de Salta y Formosa y perteneciente a la ecorregión Chaco Seco (Mereles et al., 2013). La especie fue colectada en las áreas semiáridas de Chaco, sobre suelos arcillosos a francos, con una débil capa de arenas eólicas superficial; hasta el momento ha sido encontrada únicamente dentro de cultivos de sorgo. El área ecológica en la que se desarrolla coincide con aquellas mencionadas por otros autores, así como su desarrollo en ecosistemas áridos (Anton, 1981; Sulekic \& Zapater, 2001).

Material examinado: Tragus australianus S. T. Blake. ARGENTINA. San Luis: Dep. Chacabuco, márgenes del Arroyo Papagayos, debajo del puente de Ruta Prov. 1, acceso a Papagayos, W Sierra Comechingones, $32^{\circ} 40^{\prime} 40^{\prime \prime} \mathrm{S} 64^{\circ} 59^{\prime} \mathrm{W}, 1100 \mathrm{~m}$ s.n.m., 14-I-2004, Pozner \& Belgrano 225 (CTES). PARAGUAY. Boquerón: Mariscal Estigarribia, Km 543, Estancia Jeroviá, $21^{\circ} 55^{\prime} 34^{\prime \prime} \mathrm{S} 60^{\circ} 42^{\prime} 40^{\prime \prime} \mathrm{W}$, 9-IV-2014, Mereles \& Soria 9942 (FCQ).

Tragus berteronianus Schult. ARGENTINA. Catamarca: Dep. Belén, Quebrada de Belén, $27^{\circ}$ 05' 11" S 66 53' 37' W, 20-II-1973, Ulibarri 334 (CTES). Formosa: Dep. Matacos, Ing. Juárez, en la reserva de las vías férreas, 14-I-1957, Burkart 20282 (CTES). Jujuy: Dep. Tilcara, Huacalera, Ruta Nacional 9, Valle del Río Grande, 2670 m s.n.m., $23^{\circ} 25^{\prime} \mathrm{S} 65^{\circ} 21^{\prime} \mathrm{W}, 6-\mathrm{II}-1995$, Deginani et al. 401 (CTES). Salta: Dep. Rivadavia, Pozo Largo, 350 m s.n.m., 16-I-1987, Saravia Toledo 1281 (CTES). Santiago del Estero: Dep. Ojo de Agua, Sierra de Sumampa, about $10 \mathrm{~km}$ E of Villa Ojo de Agua, 


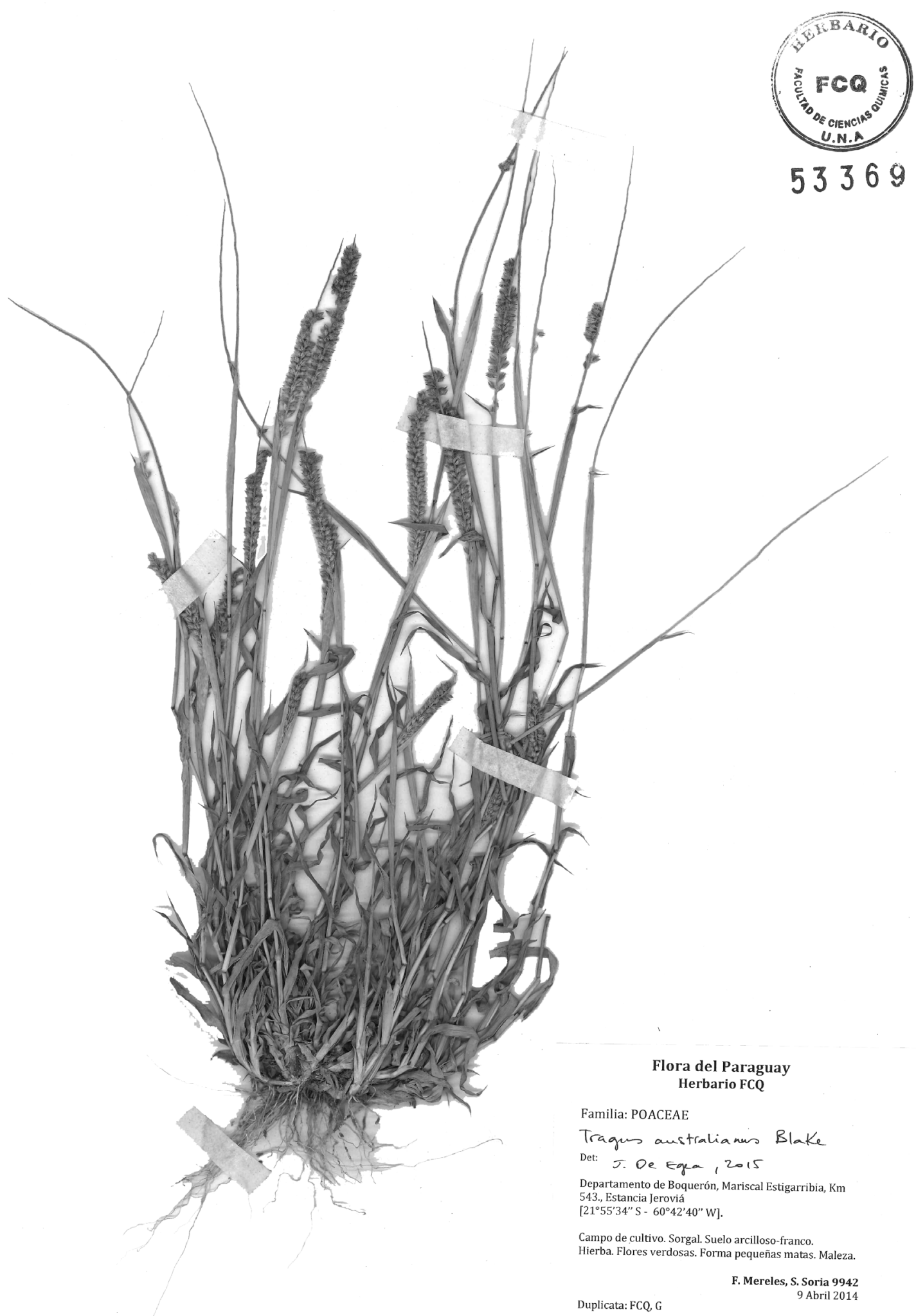

Fig. 1. Tragus australianus. Fotografía del ejemplar de herbario colectado en el departamento Boquerón (Mereles \& Soria 9942, FCQ). 


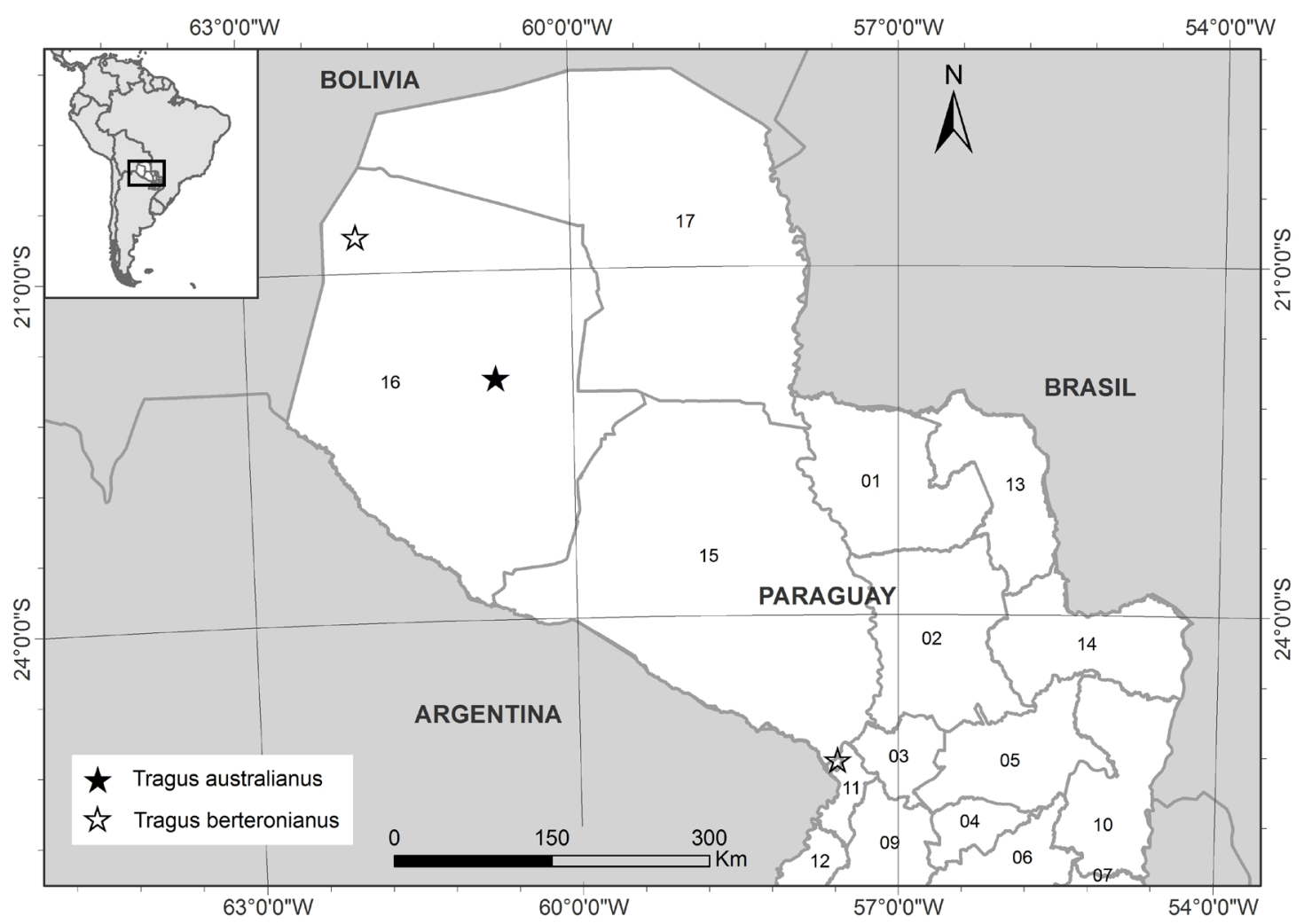

Fig. 2. Localización de los registros del género Tragus en el Paraguay.

4-III-1989, Pedersen 15159 (CTES). PARAGUAY.

Boquerón: National Park Médanos del Chaco, $20^{\circ}$ 41'03" S 61 57' 37' W, 12-XII-1998, Zardini \& Duarte 49677 (AS, MO). Capital: Asunción, Jardín Botánico, 22 $24^{\prime} 36^{\prime \prime} \mathrm{S} 55^{\circ} 36^{\prime} 43^{\prime}$ W, s/f, Rojas 7176 (MO). PERÚ. Cajamarca: Prov. Celendin, BalsasCelendin road, 1-5 Km from Balsas, Río Marañon valley, 910-1160 m s.n.m., $06^{\circ} 50^{\prime} \mathrm{S} 78^{\circ} 42^{\prime} \mathrm{W}, 23-\mathrm{II}-$ 1984, Smith 6153 (CTES).

\section{Agradecimientos}

Agradecemos especialmente a Monsanto, InBio y Dekalpar, donantes del proyecto "Actualización del conocimiento sobre malezas del Paraguay", que permitieron la realización de los trabajos de campo y las colectas. Agradecemos también al equipo de trabajo de los herbarios AS, CTES y FCQ, que recibieron a las investigadoras durante la revisión de los ejemplares con la hospitalidad de siempre.

\section{Bibliografía}

ANTON, A. M.1981. The genus Tragus (Gramineae). Kew Bull. 36: 55-61.

CLAYTON, W. D. \& F. R. RICHARDSON. 1973. Studies in The Gramineae XXXII. Kew Bull. 28: $37-48$.

CLAYTON, W. D., M. S. VORONTSOVA, K. T. HARMAN \& H. WILLIAMSON. 2014. GrassBase - The Online World Grass Flora. http://www.kew. org/data/grasses-db.html. [consultado 05 Octubre 2015].

MERELES, F., J. L. CARTES, R. P. CLAY, P. CACCIALI, C. PARADEDA, O. RODAS \& A. YANOSKY. 2013. Análisis cualitativo para la definición de las ecorregiones de Paraguay occidental. Paraquaria Nat. 1: 12-20.

PETERSON, P. M., K. ROMASCHENKO \& G. JOHNSON. 2010. A classification of the Chloridoideae (Poaceae) based on multi-gene phylogenetic trees. Molec. Phylogen. Evol. 55 (2): 580-598. doi:10.1016/j.ympev.2010.01.018

SORENG, R.J.,P.M.PETERSON,K.ROMASCHENKO, G. DAVIDSE, F. O. ZULOAGA, F. O., E. J. 
JUDZIEWICZ, T. S. FILGUEIRAS, J. I. DAVIS \& O. MORRONE. 2015. A worldwide phylogenetic classification of the Poaceae (Gramineae). J. Syst. Evol. 53: 117-137. doi: 10.1111 jse. 12150

SULEKIC, A. A. \& M. A. ZAPATER. 2001. El género Tragus (Poaceae, Zoysieae) en la Argentina. Darwiniana 39: 247-254.

TROPICOS.ORG. [permanentemente actualizado, consultado 06 Octubre 2015] Missouri Botanical Garden. <http://www.tropicos.org/ Specimen/229824>

ZULOAGA, F. O. \& M. J. BELGRANO (eds.). 2014. Catálogo de las Plantas Vasculares del Cono Sur. http://www.darwin.edu.ar/Proyectos/ FloraArgentina/FA.htm. [consultado 05 Octubre 2015]

Original recibido el 22 de enero de 2016; aceptado el 2 de mayo de 2016. 
